\title{
Do all schizophrenia patients need antipsychotic treatment continuously throughout their lifetime? A 20-year longitudinal study
}

\author{
M. Harrow*, T. H. Jobe and R. N. Faull \\ Department of Psychiatry, University of Illinois College of Medicine, Chicago, IL, USA
}

Background. The prevailing standard of care in the field involves background assumptions about the importance of prolonged use of antipsychotic medications for all schizophrenia (SZ) patients. However, do all SZ patients need antipsychotics indefinitely? Are there factors that help to identify which SZ patients can enter into prolonged periods of recovery without antipsychotics? This 20-year longitudinal research studied these issues.

Method. A total of 139 early young psychotic patients from the Chicago Follow-up Study, including 70 patients with SZ syndromes and 69 with mood disorders, were assessed, prospectively, at the acute phase and then followed up six times over the next 20 years. Patients were assessed with standardized instruments for major symptoms, psychosocial functioning, personality, attitudinal variables, neurocognition and treatment.

Results. At each follow-up, 30-40\% of SZ patients were no longer on antipsychotics. Starting at the 4.5-year follow-ups and continuing thereafter, SZ patients not on antipsychotics for prolonged periods were significantly less likely to be psychotic and experienced more periods of recovery; they also had more favorable risk and protective factors. SZ patients off antipsychotics for prolonged periods did not relapse more frequently.

Conclusions. The data indicate that not all SZ patients need treatment with antipsychotics continuously throughout their lives. SZ patients not on antipsychotics for prolonged periods are a self-selected group with better internal resources associated with greater resiliency. They have better prognostic factors, better pre-morbid developmental achievements, less vulnerability to anxiety, better neurocognitive skills, less vulnerability to psychosis and experience more periods of recovery.

Received 1 November 2011; Revised 17 January 2012; Accepted 23 January 2012; First published online 17 February 2012

Key words: Antipsychotic medications, chronic psychiatric illness, delusions, longitudinal, outcome studies, psychosis, recovery, schizophrenia.

\section{Introduction}

Do all patients with schizophrenia (SZ) need to be treated with antipsychotic medications continuously throughout their lives? Prolonged use of antipsychotic medications is the current standard of care in the field and is viewed as the cornerstone of treatment for SZ (Gilbert et al. 1995; Bosveld-van Haandel et al. 2001; Buchanan et al. 2010). This involves billions of dollars in treatment expenditures. However, some research and formulations about patients with SZ have raised empirical and theoretical issues that question the importance of continuous multi-year antipsychotic medication for all SZ (Bleuler, 1978; Fenton \& McGlashan, 1987; Harrison et al. 2001; American Diabetes Association, 2004; Harrow et al. 2005, 2007;

\footnotetext{
* Address for correspondence: M. Harrow, Ph.D., University of Illinois at Chicago, Department of Psychiatry, $1601 \mathrm{~W}$. Taylor (M/C 912), PI, Rm. 445, Chicago, IL 60612, USA.

(Email: Mharrow@psych.uic.edu)
}

Jobe \& Harrow, 2005; Lieberman et al. 2005; Menezes et al. 2006; Samaha et al. 2007; Webber \& Marder, 2008; Moncrieff, 2009; Moncrieff \& Leo, 2010; Remington \& Kapur, 2010; Ho et al. 2011).

In addition, our research, using 15-year longitudinal data from our Chicago Follow-up Study and multiple assessments at systematic intervals, has indicated that, after early antipsychotic medication treatment during the first few years of disorder, a number of SZ patients can function adequately over a prolonged period without antipsychotics (Harrow et al. 2005, 2007; Jobe \& Harrow, 2010). Research studies of other prominent investigators are in agreement (Bleuler, 1978; Fenton \& McGlashan, 1987).

Overall, based on numerous double-blind studies, there is strong evidence on the efficacy of first- and second-generation antipsychotic medications over the short term in the treatment of SZ (Gilbert et al. 1995; Buchanan et al. 2010) and there is debate about whether very early treatment improves long-term outcome 
(Owens et al. 2010). Many comprehensive treatment summaries impacting on the long-term course of SZ consider multiple treatment alternatives but do not consider the possibility of no antipsychotic medications (Tandon et al. 2008; Kreyenbuhl et al. 2010).

The importance of research into the long-term efficacy of antipsychotics and the lack of longitudinal data on its effect on outcome and potential relapse were emphasized by the President's New Freedom Commission on Mental Health (2003), noting the dearth of information on the long-term use of medications for severe mental illness.

The current research report uses longitudinal data involving a prospective assessment at index hospitalization and six subsequent follow-ups over 20 years to study whether long-term antipsychotic medication use is necessary for all SZ. It provides data on whether subgroups of SZ can experience prolonged periods of recovery without antipsychotics, and whether select risk and protective factors alter the chances of SZ going off antipsychotic medications for prolonged periods (Harrow \& Jobe, 2007). Overall, the current 20-year prospectively designed longitudinal research provides data to help answer the following questions:

(1) Is there a subgroup of SZ who, years after the acute phase of hospitalization, function adequately for a prolonged period when not receiving antipsychotic medications?

(2) Do SZ patients not in treatment with antipsychotics for a prolonged period have a high rate of relapse?

(3) What patient characteristics, including select risk and protective factors associated with more favorable outcome in SZ, identify patients who may go off antipsychotic medications for a prolonged period.

\section{Method}

\section{Patient sample}

The current investigation was based on data from the Chicago Follow-up Study, a prospectively designed, longitudinal, multi-follow-up research program studying major symptoms and functioning, outcome and recovery in SZ and other psychotic disorders (Grinker \& Harrow, 1987; Harrow et al. 1990, 2000, 2005, 2009; Carone et al. 1991; Jobe \& Harrow, 2005, 2010 ; Harrow \& Jobe, 2007, 2010; Bonner-Jackson et al. 2010). Beginning relatively early in their illness, 139 young patients were studied prospectively at the acute phase of hospitalization and then reassessed at six subsequent follow-ups over a 20 -year period. The followups occurred at 2, 4.5, 7.5, 10, 15 and 20 years post-index hospitalization.
The sample of 139 DSM-III diagnosed patients included 70 patients with SZ syndromes (61 SZ patients and nine schizo-affective patients) and a control sample of 69 patients with mood disorders who were psychotic at index hospitalization. All SZ met the 6 months' duration of illness criterion (none were schizophreniform patients). From among the 70 SZ, 59 were assessed at the 20-year follow-ups. The other 11 SZ were assessed at each of the first five follow-ups, including the 15-year follow-ups. The 69 non-SZ patients who were psychotic at index included 38 psychotic bipolar patients and 31 psychotic unipolar depressives.

After Institutional Review Board approval, signed informed consent was obtained from all subjects. The research diagnoses were based on structured research interviews conducted during index hospitalization (Grinker \& Harrow, 1987), supplemented by intake interviews at hospital admission. Satisfactory interrater reliability was established for diagnosis (e.g. a $\kappa$ value of 0.88 for SZ).

At index hospitalization, the patients were consecutive admissions at two Chicago hospitals (a private hospital and a state hospital) within the limitation of giving preference to younger patients (aged between 17 and 32 years at index) with no or fewer previous hospitalizations. Forty-one percent of the sample were first-admission patients at index, and another $25 \%$ had only one previous hospitalization. The mean age of the patients was 22.9 years at index hospitalization. The diagnostic groups did not differ in age. The mean level of education at index was 13.0 years. Fifty-two percent of the sample was male, with more male SZ patients and more female mooddisordered psychotic patients.

\section{Follow-up assessments}

Trained interviewers who were not informed of diagnoses or the results of previous assessments conducted the follow-ups. We used structured interviews [the Schedule for Affective Disorders and Schizophrenia (SADS) and a functioning interview (Endicott \& Spitzer, 1978; Grinker \& Harrow, 1987; Harrow et al. 2005)] to evaluate psychosis (the presence of delusions and/or hallucinations during the follow-up year) and other major symptoms (negative symptoms, anxiety and affective symptoms). We also used these instruments to assess global outcome and self-support, social functioning, work functioning, rehospitalization and treatment (Grinker \& Harrow, 1987; Strauss \& Carpenter, 1972). The measure of global outcome that includes symptoms and life functioning is the eightpoint Levenstein-Klein-Pollack (LKP) scale, used in many previous reports (Levenstein et al. 1966; Grinker 
Table 1. Patients with schizophrenia (SZ) on antipsychotic medications at each assessment over 20 years

\begin{tabular}{|c|c|c|c|c|}
\hline $\begin{array}{l}\text { Follow-up } \\
\text { (years) }\end{array}$ & $\begin{array}{l}\text { Antipsychotic } \\
\text { medications } \\
(\%)\end{array}$ & $\begin{array}{l}\text { Other psychiatric } \\
\text { medications } \\
\text { (no antipsychotics) } \\
(\%)\end{array}$ & $\begin{array}{l}\text { In treatment } \\
\text { (no medications) } \\
(\%)\end{array}$ & $\begin{array}{l}\text { No mental } \\
\text { health treatment } \\
(\%)\end{array}$ \\
\hline 2 & 68 & 5 & 11 & 18 \\
\hline 4.5 & 66 & 9 & 5 & 20 \\
\hline 7.5 & 63 & 14 & 2 & 22 \\
\hline 10 & 62 & 10 & 3 & 25 \\
\hline 15 & 66 & 3 & 5 & 26 \\
\hline 20 & 62 & 9 & 5 & 24 \\
\hline
\end{tabular}

\& Harrow, 1987; Harrow et al. 1990, 2000, 2005; Harrow \& Jobe, 2007) We also administered measures to assess potential risk and protective factors. These included cognitive tests to evaluate accessing of stored information (Wechsler, 1955) and abstract ability (Marengo et al. 1980), although cognitive assessments, including executive functioning, involve multiple skills, often with complex relationships to major symptoms (Reed et al. 2002; Dibben et al. 2009). We used questionnaires to assess attitudinal and personality variables, including anxiety at each of the six assessments (Katz \& Lyerly, 1963). We also used two major scales involving risk and protective factors that have theoretical relevance and that our team and other investigators have found relate to outcome in SZ. These are scales of prognostic variables reported by Vaillant (1978) and Stephens et al. (1997) and a scale by Zigler and co-workers assessing pre-morbid developmental achievement (Zigler \& Levine, 1983; Zigler \& Glick, 2001).

Information on treatment and outcome at the 15- and/or 20-year follow-ups was available for $73 \%$ of the original sample assessed. The 70 patients with SZ did not differ significantly on major demographic variables from a subsample of SZ who were assessed at index hospitalization and at the first follow-ups, but not assessed at the 15- or 20-year follow-ups.

\section{Operational definition of recovery}

The criteria for global 'recovery' during a follow-up year, defined operationally, required no positive or negative symptoms, and no rehospitalizations during the follow-up year. Also required were partially adequate (or better) work and social functioning (scores of $\geqslant 2$ on the Strauss-Carpenter scales; Strauss \& Carpenter, 1972). Recovery at any follow-up does not automatically prejudge whether recovery will continue during future years, which may be a function of $(a)$ the natural course of SZ, $(b)$ individual characteristics of the patient assessed, and $(c)$ treatment.

\section{Medications}

Table 1 reports data on the percentage of SZ on medications at each follow-up over 20 years. As frequently found in the natural course of a large series of SZ, there was no single, uniform treatment plan that applied to all patients. Rather, at the 20-year followups, $62 \%$ of the SZ were on antipsychotic medications with or without other medications; $79 \%$ of the SZ on antipsychotics at the 20-year follow-ups had been on an antipsychotic at the 2-year follow-ups. At the 20-year follow-ups, $28 \%$ of the patients with other types of psychotic disorders were on antipsychotics with or without other medications, and an additional $37 \%$ were on other psychiatric medications but not on antipsychotics.

\section{SZ not on antipsychotic medications for prolonged periods}

Although the medication course for many SZ varied over the 20 years, 24 of the $70 \mathrm{SZ}$ were prescribed antipsychotics at every one of the follow-up assessments over 20 years, and 15 SZ were not on antipsychotic medications at any one of the six assessments. These two groups of SZ, $(a)$ one group prescribed antipsychotic medications throughout the 20-year periods and $(b)$ another group not on antipsychotics throughout the past 18 years of the 20-year period, represent important subgroups. They allow study of whether a group of SZ who are medication free for many successive years can show prolonged periods of recovery. They also provide an opportunity to determine outcome for SZ prescribed antipsychotics for many successive years. Longitudinal data from 


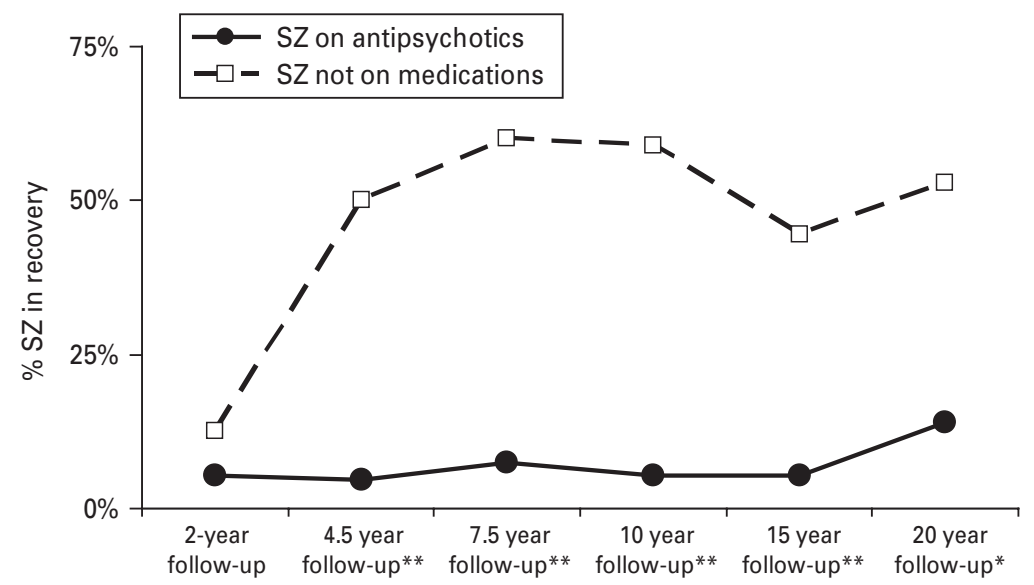

Fig. 1. Relationship between recovery and use of antipsychotics in schizophrenia (SZ). ${ }^{*} p<0.01,{ }^{* *} p<0.001$.

both groups have previously been unavailable to the field.

\section{Results}

Frequency of antipsychotic treatment and group differences in psychosis and recovery

By the 2-year follow-ups almost $35 \%$ of the SZ were no longer taking antipsychotic medications. Although the specific SZ not an antipsychotics shifted somewhat from follow-up to follow-up, this figure of about 35\% remained relatively constant for the 20 years of followups.

At the 2-year assessment there were no significant differences in severity of psychosis between SZ on antipsychotic medications and SZ not on any medications. However, starting at the 4.5-year follow-ups and continuing over the next 15 years, the SZ who were not on antipsychotic medications were significantly less psychotic than those on antipsychotics $(p<0.05)$. The $\chi^{2}$ values with $1 \mathrm{df}$ varied from 4.78 to 13.59 , with three of the five $\chi^{2}>10.0(p<0.002)$.

In addition (see Fig. 1), starting at the 4.5-year follow-up and continuing at each assessment over the next 15 years, a significantly larger percentage of SZ not on medications experienced a period of recovery $(p<0.01)$, which also requires adequate work and social functioning. The $\chi^{2}$ values with $1 \mathrm{df}$ varied from 9.06 to 19.75 , with all $p<0.01$.

\section{Periods of recovery: a cumulative analysis of recovery in SZ not on antipsychotic medications over 20 years}

Of those SZ on antipsychotic medications continuously over all follow-ups (24 SZ) over the 15-20-year period, only four $(17 \%)$ ever entered into a period meeting the operational definition of recovery during any of the six follow-ups. The two factors most prominent in these SZ on antipsychotics who did not meet the operational definition of recovery were $(a)$ the presence of psychosis, and $(b)$ not working. By contrast, of the $15 \mathrm{SZ}$ who had gone off antipsychotic medications prior to the 2-year follow-up assessments and subsequently were not on antipsychotics at any of the six follow-ups over the 20-year period, the great majority $(13 / 15$ or $87 \%)$ experienced two or more periods of recovery.

Relationship between antipsychotic use and recovery for patients who had mood disorders with psychosis at index hospitalization

When both (a) global functioning and (b) psychosis scores for the psychotic mood-disordered patients were compared to the SZ during the 20 years of follow-ups, there were strong significant outcome differences in favor of the mood-disordered patients. For comparison of SZ with originally psychotic mooddisordered patients on global outcome, a $2 \times 6$ mixed design, repeated-measures ANOVA (two diagnostic groups and six follow-up assessments) showed large significant differences $(F=11.12, \mathrm{df}=1,65, p=0.001)$. Similarly, a $2 \times 6$ repeated-measures ANOVA for psychosis, comparing SZ and mood-disordered patients, showed large significant differences $(F=11.22$, $\mathrm{df}=1,50, p=0.002)$. The assessment of psychotic patients who had experienced mood disorders focused on a comparison of patients on antipsychotic medications versus those not on any psychiatric medications. At every follow-up assessment over the 20 years, including the 2-year follow-ups, significantly more of the unmedicated patients than those on antipsychotics experienced a period of recovery. The $\chi^{2}$ values, with $1 \mathrm{df}$, varied from 3.71 to $20.22(p<0.05)$. 


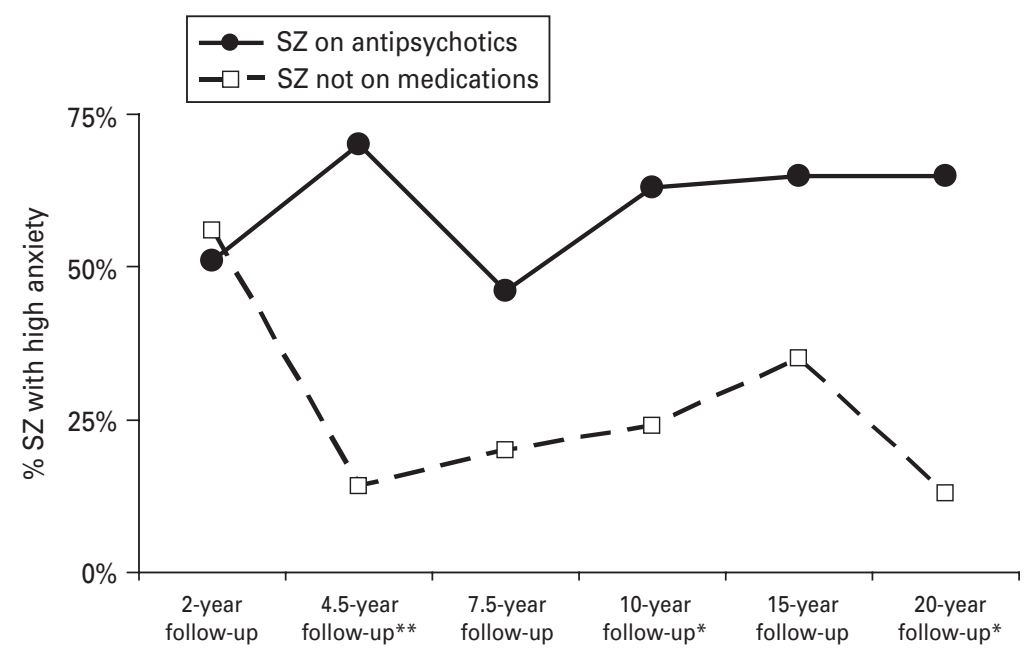

Fig. 2. Relationship between vulnerability to anxiety and use of antipsychotic medications in schizophrenia (SZ). ${ }^{*} p<0.01$, ** $p<0.001$.

Risk and protective factors: pre-morbid and early prognostic characteristics that separate SZ who stay on antipsychotics versus those not on medications for prolonged periods

Analysis was conducted of potential risk and protective factors that separate the SZ taking antipsychotic medications from those who had removed themselves or been removed from antipsychotic treatment. The data on SZ who have good and poor prognostic features, and who have good and poor pre-morbid developmental achievements, point to different characteristics of the SZ who are more likely to remain on antipsychotic medications versus those who do not remain on antipsychotic medications. The SZ who go off antipsychotic medications for a prolonged period are a self-selected group. These two prognostic type indices are based on pre-morbid features and on prognostic characteristics present at index hospitalization or before, and both were collected long before any of the 20 years of follow-ups. Starting at the 2-year followups, more of the SZ with poor prognostic features and poor developmental achievements were on antipsychotics, with these differences between SZ with good and poor features being significant $(p<0.02)$ in seven of the 12 comparisons over the 20-year period $\left(\chi^{2}\right.$ varied from 5.77 to 10.15$)$, and additionally showing a trend $(p<0.09$ and $p<0.08)$ in another two of the 12 comparisons.

Previously reported research by our group and other research on early impaired social functioning and psychosis provide evidence that a series of early prognostic characteristics are important predictors of potential periods of recovery and of less vulnerability to chronic psychosis (Jones et al. 1994; Harrow \& Jobe, 2007, 2010; White et al. 2009; Cornblatt et al. 2011).
Differences in vulnerability to anxiety between SZ on antipsychotic medications and unmedicated SZ

Data on anxiety are reported in Fig. 2. After the 2-year follow-ups, starting at the 4.5-year follow-ups, SZ who are high on anxiety or get anxious more easily were more likely to remain on antipsychotic medications. Thus, at the 4.5-year follow-ups $\left(\chi^{2}=13.04,1 \mathrm{df}\right.$, $p<0.001)$, the 10-year follow-ups $\left(\chi^{2}=7.08,1 \mathrm{df}\right.$, $p<0.001)$ and the 20-year follow-ups $\left(\chi^{2}=11.88\right.$, $1 \mathrm{df}, p<0.001)$, SZ on antipsychotic medications were significantly more anxious than those on no medications. Akathisia may also be involved. Previous research has provided evidence that less anxious SZ have better longitudinal courses (Harrow et al. 2008; Jobe \& Harrow, 2010).

\section{Neurocognitive differences: SZ on antipsychotics versus SZ on no medications}

Potential differences in neurocognitive skills between SZ who stay on antipsychotics and those who remove themselves or are removed from antipsychotic medications were explored, using two cognitive tests. The first was a test of skills in accessing general information: the Wechsler Adult Intelligence Scale (WAIS), Information Test (Wechsler, 1955). The SZ not on antipsychotic medications showed significantly better cognitive skill in accessing stored knowledge than the SZ on antipsychotics at three of the six follow-ups. The $t$ values ranged from 2.04 to $2.62(p<0.05)$ with non-significant trends ( $p=0.09$ to $p=0.13$ ) towards better cognitive functioning for SZ not on antipsychotics on the other three follow-ups.

Data on successful abstraction are reported in Fig. 3. Using the Proverbs Test (Gorham, 1956) to assess 


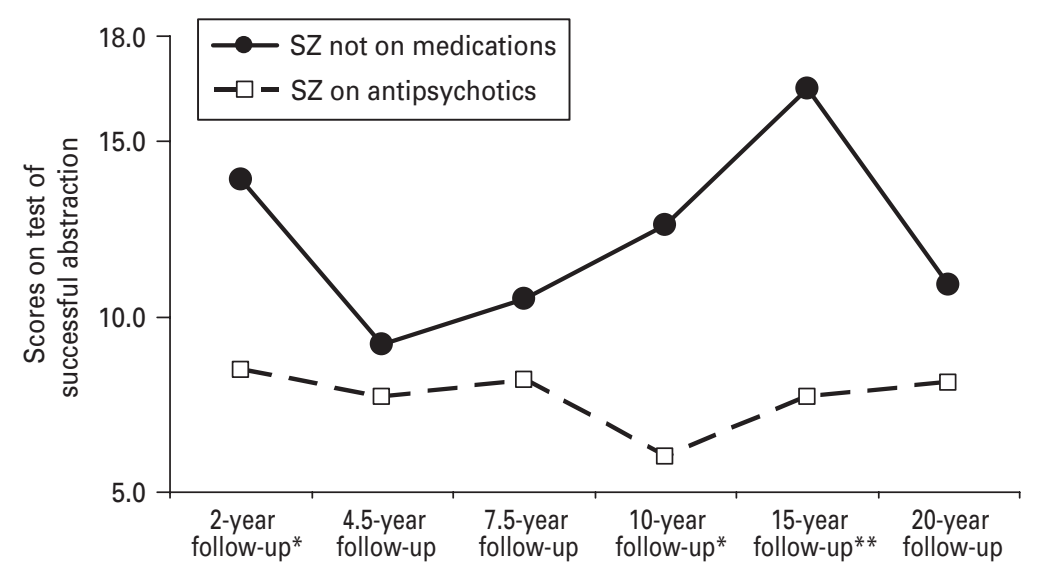

Fig. 3. Cognition scores on test of successful abstraction for schizophrenia (SZ). ${ }^{*} p<0.05,{ }^{* *} p<0.01$.

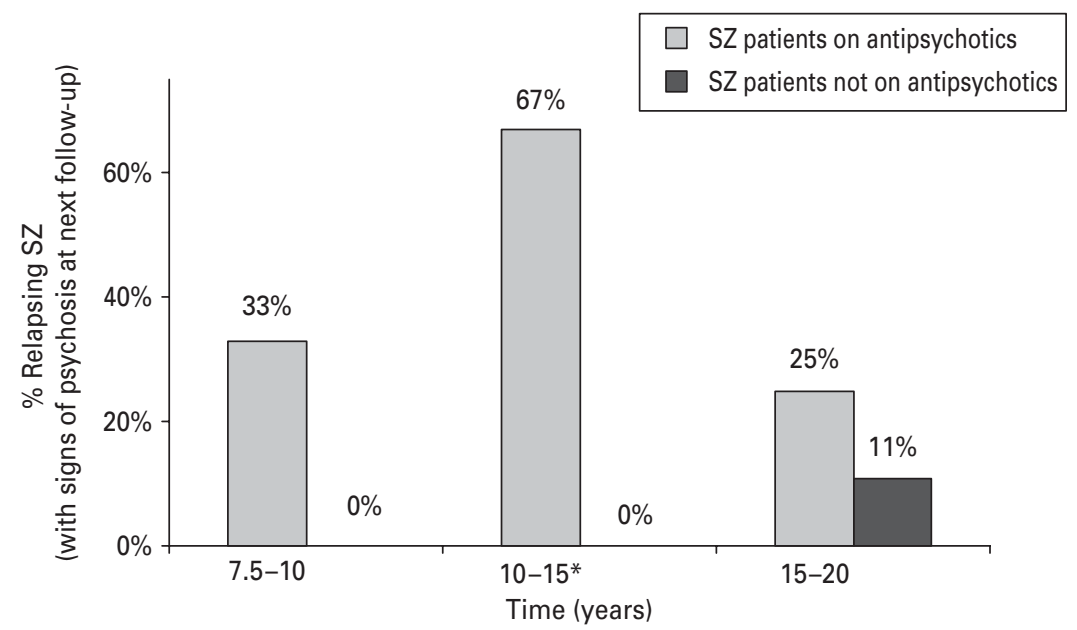

Fig. 4. How protective are antipsychotics against relapse? ${ }^{*} p<0.01$.

successful abstraction ability at each follow-up assessment, the SZ not on antipsychotic medications also showed significantly better abstract skills at three of the six follow-ups. The $t$ values ranged from 2.35 to $3.47(p<0.03)$. Previous research of ours and others has provided evidence that SZ with poorer neurocognitive performance have poorer long-term outcomes (Bonner-Jackson et al. 2010). These data suggest that assessment of other types of cognitive skills would be profitable.

\section{Potential relapse of SZ patients not on antipsychotic medications}

Potential relapse of SZ on or off antipsychotic medications has always been a major issue. A discriminating assessment of potential relapse involves analyses at a succeeding follow-up of the group of SZ who were on antipsychotic medications and were not psychotic at the previous follow-up, and comparing them with the group of SZ who were not on antipsychotics who were not psychotic at the previous follow-up. This important analysis involves a comparison of the relapse rate of those two groups of SZ, with both groups composed of SZ who were not psychotic at the previous follow-up.

Figure 4 reports data on relapse for the two groups. After the 2-year follow-ups, the unmedicated SZ who were not psychotic at the previous follow-up had low rates of relapse at the next follow-ups. At each of the five comparison points, the rate of psychotic relapse for SZ who were not psychotic at a previous follow-up was lower for SZ not on medications than for SZ who were on antipsychotic medications, with differences significant at the 15-year follow-ups $\left(\chi^{2}=8.18,1 \mathrm{df}\right.$, $p<0.01)$.

\section{Discussion}

The central issue posed is whether all SZ need to be treated with antipsychotic medications continuously 
throughout their lives, an issue that also involves costs of billions of dollars of expenditures, and multiple potential side-effects. The efficacy and effectiveness of antipsychotic medications in reducing psychosis during the acute phase, especially flagrant psychosis, has clearly been established. Some of this reduction of flagrant psychosis to mild levels of psychosis for most, and no psychosis for some, may be related to its sedating effect as a 'major tranquilizer'. Its long-term use as a maintenance medication in SZ is the current standard of care. However, despite many short-term (0-2 years) double-blind studies, long-term evaluations based on multi-year assessments at systematic intervals that include SZ who have left treatment are rare, with systematic longitudinal data on this issue unavailable to the field.

The current data, based on 20 years of assessments, indicate that not all SZ need antipsychotic medications continuously. Of the $15 \mathrm{SZ}$ who had gone off antipsychotic medications prior to the 2-year follow-up assessments and subsequently were not on antipsychotics for the next 18 years, the great majority $(87 \%)$ experienced at least two periods of recovery. Most of these SZ were not permanently 'cured' but the post-hospital course and outcome of this subgroup of $15 \mathrm{SZ}$ was significantly better $(p<0.001)$ than the SZ on antipsychotics throughout the 20 years. Other aspects of our data suggest that internal factors associated with their greater resiliency was one of the prominent factors linked to their better course and outcome.

By contrast, the great majority of SZ on antipsychotics continuously did not show any sustained periods of recovery. Several major investigators have noted that antipsychotic medications are, at best, only partially effective treatments (Carpenter et al. 2003) with only a low rate of complete recovery in treated SZ (Ventura et al. 2011).

These results on the better functioning of unmedicated SZ were influenced by some patients who had started to recover from their symptoms leaving treatment because of their improved status, and other SZ improving after leaving treatment.

\section{Are there many SZ who are not in antipsychotic treatment?}

The better functioning of SZ not on antipsychotics, on a longitudinal basis after the first 4 years, raises the issue of whether the group of SZ not in antipsychotic treatment represent (a) a moderate-sized (or larger) sample of SZ or (b) only a few SZ. Our 20-year data indicate that the subsample of SZ not on antipsychotics represent a moderate-sized group (30-40\%) of SZ.

\section{The issue of relapse}

Another issue concerns the potential relapse of SZ who, at an earlier follow-up, were psychosis free. When SZ are not on antipsychotic medications for a prolonged period, and at the time are not psychotic, are they likely to be psychotic 5 years later? The data indicate that many of these unmedicated SZ patients have good internal resources and are those who are less vulnerable to psychosis. The data in Fig. 4 indicate that these SZ are more likely to manifest a very low rate of relapse when off antipsychotics for a prolonged period.

For most SZ not on medications or not in treatment this was their choice, at times against professional advice. For professionals engaged in treatment, removing an SZ from medication that he/she had been treated with over a prolonged period often involves a difficult decision because of concerns (with possible legal implications) that an SZ doing well may experience a psychotic relapse. However, continuous exposure to antipsychotics poses risks, and the current data indicate that many SZ continuing on antipsychotics also relapse (see Fig. 4), posing a delicate risk-benefit assessment for the clinician.

\section{The poorer functioning of the patients with mood disorders who were treated with antipsychotic medications}

Not surprisingly, psychotic mood-disordered patients had better global outcomes and less psychosis than the SZ $(p<0.01)$. However, the 20-year data on poorer functioning for the patients with mood disorders who were on antipsychotics are consistent with the data on the poorer long-term functioning of the SZ who were on antipsychotic medications. These data on mooddisordered patients are also in accord with earlier reports of ours and of other major investigators, years ago, that began to question extremely optimistic views on lithium as a miracle cure for $70-80 \%$ of bipolar patients (Harrow et al. 1990; Goldberg \& Harrow, 1999).

\section{Periods of recovery}

Recovery is often viewed as a static variable, indicating multiple years of good outcome. However, we should adopt a more realistic outlook toward recovery in severe mental disorders. Rather than viewing recovery as a permanent state, we should view it in terms of periods of recovery with its length partly related to the extent of the underlying biological vulnerability to psychosis of the SZ. For some of these patients, recovery may last for a relatively short period, such as 1-3 years, and for some patients, 
periods of recovery may last over 10 years, or even for a lifetime.

\section{The prediction of periods of recovery in SZ}

Fitting with the multi-dimensional nature of SZ, recovery in SZ is a multi-dimensional process, with many factors involved; there are also many different variables that show some potential to predict aspects of outcome in this disorder. This includes a series of prognostic variables in addition to risk factors (e.g. vulnerability to anxiety) and protective factors such as favorable pre-morbid developmental achievements, better neurocognitive function and even some attitudinal variables (Harrow et al. 2005, 2008, 2009; Harrow \& Jobe, 2007; Jobe \& Harrow, 2010).

\section{Towards a more representative view of outcome in modern-day SZ}

Treatment is very important, especially for those patients with SZ who are vulnerable to frequent acute disorder and chronic positive symptoms. However, many other factors are involved in outcome and recovery. The SZ underlying diathesis, which includes a vulnerability to psychosis and other psychopathology, is a major factor in their disorder and in potential chronicity for many SZ. This underlying vulnerability accounts for much of the chronic or partially chronic picture found in 50-65\% of SZ (Harrow et al. 2005; Harrow \& Jobe, 2010). We might view SZ as involving a biological vulnerability to sustained psychosis, to other major symptoms and to functional disability, with various biological and non-biological risk and protective factors (e.g. vulnerability to anxiety, neurocognitive impairment, hardiness-resiliency, personality-attitudinal factors, and numerous other factors) influencing whether the underlying vulnerability will manifest itself and result in sustained episodes of SZ for some (but not all) of these biologically vulnerable people. Some of these risk and protective factors may be endophenotypes (Gottesman \& Shields, 1982; Gur et al. 2007; Kendler \& Neale, 2010). As one example, many types of neurocognitive impairment in SZ are familial and show a genetic influence, and our own data indicate that neurocognitive domains are longitudinally stable (trait-like) across broad domains in SZ (Burdick et al. 2006), thus meeting two of the characteristics for endophenotypes (heritability and stability). Our longitudinal data suggest that SZ internal resources are as important as their treatment.

It is important to note that lack of adherence with medication schedules can interfere with favorable outcome for some medicated patients. However, lack of adherence does not account at all for the relatively favorable outcome of a number of untreated SZ.

As in many other areas of medicine, we in the field have considerable contact with chronic or partially chronic patients in aftercare treatment. We do not see most of the SZ not in treatment. Much of our impressions about outcome in SZ are based on chronic patients who do come for treatment and who we see frequently. The clinic SZ patients do not give us a complete picture of outcome for all SZ. These relapsing patients are the ones from whom opinions are formed about the necessity for continuous antipsychotic medications throughout all SZ lifetimes. Cohen \& Cohen (1984) have written about the bias we can easily develop from our frequent contact with these clinic patients.

\section{Major issues concerning long-term treatment with antipsychotic medications}

Two major issues concerning long-term treatment with antipsychotic medications are: (1) do all SZ patients need to be on antipsychotics throughout their lives, and (2) is very long-term treatment with antipsychotic medications undesirable? Regarding the first question, the current results provide strong evidence that not all SZ need to be on antipsychotic medications for all of their lives. The data point to risk and protective factors. If protective factors are present and the SZ has already shown some periods of recovery, and wants to try a period without antipsychotics, then he/she is a good candidate to try going off antipsychotics, although, as with many other medical procedures, there is no certainty of the results.

The data suggest that the main factor leading to the more favorable outcome of SZ not on antipsychotics for a prolonged period is the leaving of treatment by SZ with good prognosis and favorable outcome who are less vulnerable to psychosis.

The other major issue is whether very long-term use of antipsychotic medications produces undesirable effects for some SZ. The current 20-year longitudinal data do not provide direct evidence on this second question. Some have proposed that, over a prolonged period of antipsychotic treatment, supersensitivity of dopamine receptors may occur as a compensation of the brain for many years of reduced dopamine resulting from dopamine blockade (Chouinard \& Jones, 1980; Samaha et al. 2007; Remington \& Kapur, 2010). The current data do not pertain directly to the dopamine supersensitivity hypothesis of continuous antipsychotic use, or to potential decreases in brain volume associated with many years of antipsychotic medication treatment (Moncrieff \& Le, 2010; Ho et al. 2011). 


\section{Have other longitudinal studies found similar results?}

Is the current subsample of untreated SZ who showed favorable outcomes unusual or unique? Several other major longitudinal studies, both in the USA and elsewhere, have found similar results on subsamples of unmedicated and untreated SZ, many of whom are functioning better then expected. Most of these studies did not involve evaluations of multiple risk and protective factors and multiple periods of assessments, as in the present research. However, they studied longitudinal data indicating that some SZ not in treatment show more favorable outcomes. Thus, longitudinal studies that clearly note and both discuss and comment on the subsamples of unmedicated and untreated SZ functioning relatively well include the Chestnut Lodge Study (Fenton \& McGlashan, 1987), a longitudinal study by Bleuler (1978), the Vermont studies of Harding et al. (1987) and the shorter-term studies of Bola \& Mosher (2002) and the Finnish NeedAdapted Program (Lehtinen et al. 2000). Other important longitudinal studies that found that their untreated SZ were functioning significantly better than SZ in treatment, years later, include the Alberta hospitals studies (Bland et al. 1978) and the important World Health Organization studies (Harrison et al. 2001).

\section{Acknowledgements}

This work was supported, in part, by United States Public Health Service (USPHS) grants MH-26341 and MH-068688 from the National Institute of Mental Health, USA (M. Harrow).

\section{Declaration of Interest}

None.

\section{References}

American DiabetesAssociation (2004). Consensus development conference on antipsychotic drugs and obesity. Diabetes Care 27, 596-601.

Bland R, Parker J, Orn H (1978). Prognosis in schizophrenia : prognostic predictors and outcome. Archives of General Psychiatry 35, 72-77.

Bleuler M (1978). The Schizophrenic Disorders: Long-Term Patient and Family Studies. Yale University Press: New Haven.

Bola J, Mosher L (2002). At issue: predicting drug-free treatment response in acute psychosis from the Soteria project. Schizophrenia Bulletin 28, 559-575.

Bonner-Jackson A, Harrow M, Rosen C (2010).

Neurocognition in schizophrenia : a 20-year multi-follow-up of the course of processing speed and stored knowledge. Comprehensive Psychiatry 51, 471-479.

Bosveld-van Haandel L, Slooff C, van den Bosch R (2001). Reasoning about the optimal duration of prophylactic antipsychotic medication in schizophrenia : evidence and arguments. Acta Psychiatrica Scandinavica 103, 335-346.

Buchanan R, Kreyenbuhl J, Kelly D, Noel J, Boggs D, Fischer B, Himelhoch S, Fang B, Peterson E, Aquino P, Keller W (2010). The 2009 schizophrenia PORT psychopharmacological treatment recommendations and summary statements. Schizophrenia Bulletin 36, 71-93.

Burdick K, Harrow M, Goldberg J (2006). Neurocognition as a stable endophenotype in bipolar disorder and schizophrenia. Journal of Nervous and Mental Disease 194, 255-267.

Carone J, Harrow M, Westermeyer J (1991). Posthospital course and outcome in schizophrenia. Archives of General Psychiatry 48, 247-253.

Carpenter Jr. WT, Appelbaum PS, Levine RJ (2003). The Declaration of Helsinki and clinical trials: a focus on placebo-controlled trials in schizophrenia. American Journal of Psychiatry 160, 356-362.

Chouinard G, Jones BD (1980). Neuroleptic-induced supersensitivity psychosis: clinical and pharmacologic characteristics. American Journal of Psychiatry 137, 16-21.

Cohen P, Cohen J (1984). The clinician's illusions. Archives of General Psychiatry 41, 1178-1182.

Cornblatt BA, Carrión RE, Addington J, Seidman L, Walker EF, Cannon TD, Cadenhead KS, McGlashan TH, Perkins DO, Tsuang MT (2011). Risk factors for psychosis: impaired social and role functioning. Schizophrenia Bulletin. Published online: 10 November 2011. doi:10.1093/schbul/ sbr136.

Dibben C, Rice C, Laws K, McKenna P (2009). Is executive impairment associated with schizophrenic syndromes? A meta-analysis. Psychological Medicine 39, 381-392.

Endicott J, Spitzer R (1978). A diagnostic interview. Archives of General Psychiatry 35, 837-844.

Fenton W, McGlashan T (1987). Sustained remission in drug-free schizophrenic patients. American Journal of Psychiatry 144, 1306-1309.

Gilbert PL, Harris MJ, McAdams LA, Jeste DV (1995). Neuroleptic withdrawal in schizophrenic patients: a review of the literature. Archives of General Psychiatry 52, 173-188.

Goldberg J, Harrow M (eds) (1999). Bipolar Disorders: Clinical Course and Outcome. American Psychiatric Press: Washington, DC.

Gorham D (1956). A proverbs test for clinical and experimental use. Psychological Reports 2, 1-12.

Gottesman I, Shields J (1982). Schizophrenia: The Epigenetic Puzzle. Cambridge University Press: New York.

Grinker R, Harrow M (eds) (1987). Clinical Research in Schizophrenia: A Multidimensional Approach. Charles C. Thomas: Springfield, IL.

Gur RE, Calkins ME, Gur RC, Horan WP, Nuechterlein KH, Seidman LJ, Stone WS (2007). The Consortium on the Genetics of Schizophrenia: neurocognitive endophenotypes. Schizophrenia Bulletin 33, 49-68. 
Harding C, Brooks G, Ashikiga T, Strauss J, Breier A (1987). The Vermont longitudinal study of persons with severe mental illness: II. Long-term outcome of subjects who retrospectively met DSM-III criteria for schizophrenia. American Journal of Psychiatry 144, 727-735.

Harrison G, Hopper K, Craig T, Laska E, Siegel C, Wanderling J, Dube K, Ganev K, Giel R, an der Heiden W, Holmberg SK, Janca A, Lee PW, Leon CA, Malhotra S, Marsella AJ, Nakane Y, Sartorius N, Shen Y, Skoda C, Thara R, Tsirkin SJ, Varma VK, Walsh D, Wiersma D (2001). Recovery from psychotic illness: a 15- and 25-year international follow-up study. British Journal of Psychiatry 178, 506-517.

Harrow M, Goldberg J, Grossman L, Meltzer H (1990). Outcome in manic disorders: a naturalistic follow-up study. Archives of General Psychiatry 47, 665-671.

Harrow M, Grossman L, Herbener E, Davis E (2000). Ten-year outcome: patients with schizoaffective disorders, schizophrenia, affective disorders, and mood-incongruent psychotic symptoms. British Journal of Psychiatry 177, 421-426.

Harrow M, Grossman L, Jobe T, Herbener E (2005). Do patients with schizophrenia ever show periods of recovery? A 15-year multi-follow-up study. Schizophrenia Bulletin 31, 723-734.

Harrow M, Hansford B, Astrachan-Fletcher E (2009). Locus of control: relation to schizophrenia, to recovery, and to depression and psychosis - a 15-year longitudinal study. Psychiatry Research 168, 186-192.

Harrow M, Jobe T (2007). Factors involved in outcome and recovery in schizophrenia patients not on antipsychotic medications: a 15-year multi-follow-up study. Journal of Nervous and Mental Disease 195, 406-414.

Harrow M, Jobe T (2010). How frequent is chronic multiyear delusional activity and recovery in schizophrenia : a 20-year multi-follow-up. Schizophrenia Bulletin 36, 192-204.

Harrow M, Jobe T, Astrachan-Fletcher E (2008). Prognosis of persecutory delusions in schizophrenia: a 20-year longitudinal study. In Persecutory Delusions: Assessment, Theory and Treatment (ed. D. Freeman, P. Garety and R. Bentall), pp. 73-90. Oxford University Press: Oxford.

Ho BC, Andreasen NC, Ziebell S, Pierson R, Magnotta V (2011). Long-term antipsychotic treatment and brain volumes: a longitudinal study of first-episode schizophrenia. Archives of General Psychiatry 68, 128-137.

Jobe T, Harrow M (2005). Long-term outcome of patients with schizophrenia : a review. Canadian Journal of Psychiatry 50, 892-900.

Jobe T, Harrow M (2010). Schizophrenia course, long-term outcome, recovery, and prognosis. Current Directions in Psychological Science 19, 220-225.

Jones P, Murray R, Rodgers B, Marmot M (1994). Child developmental risk factors for adult schizophrenia in the British 1946 birth cohort. Lancet 344, 1398-1402.

Katz M, Lyerly S (1963). Methods for measuring adjustment and social behavior in the community: I. Rationale, description, discriminative, validity and scale development. Psychological Reports 13, 503-535.

Kendler K, Neale M (2010). Endophenotype: a conceptual analysis. Molecular Psychiatry 15, 789-797.
Kreyenbuhl J, Buchanan R, Dickerson F, Dixon L (2010). The Schizophrenia Patient Outcomes Research Team (PORT): updated treatment recommendations 2009. Schizophrenia Bulletin 36, 94-103.

Lehtinen V, Aaltonen J, Koffert T, Räkköläinen V, Syvälahti E (2000). Two-year outcome in first-episode psychosis treated according to an integrated model. Is immediate neuroleptisation always needed? European Psychiatry 15, 312-320.

Levenstein D, Klein D, Pollack M (1966). Follow-up study of formerly hospitalized voluntary psychiatric patients: the first two years. American Journal of Psychiatry 122, 1102-1109.

Lieberman J, Stroup S, McEvoy J, Swartz M, Rosenheck R, Perkins D, Keefe R, Davis S, Davis C, Lebowitz B, Severe J, Hsiao J (2005). Effectiveness of antipsychotic drugs in patients with chronic schizophrenia. New England Journal of Medicine 353, 1209-1223.

Marengo J, Harrow M, Rogers C (1980). A Manual for Scoring Abstract and Concrete Responses to the Proverbs Test. ASIS/NAPS No. 03646. Microfiche Publications: New York.

Menezes N, Arenovich T, Zipursky R (2006). A systematic review of longitudinal outcome studies of first-episode psychosis. Psychological Medicine 36, 1349-1362.

Moncrieff J (2009). A critique of the dopamine hypothesis of schizophrenia and psychosis. Harvard Review of Psychiatry 17, 214-225.

Moncrieff J, Leo J (2010). A systematic review of the effects of antipsychotic drugs on brain volume. Psychological Medicine 40, 1409-1422.

Owens DC, Johnstone EC, Miller P, Macmillan JF, Crow TJ (2010). Duration of untreated illness and outcome in schizophrenia: test of predictions in relation to relapse risk. British Journal of Psychiatry 196, 296-301.

President's New Freedom Commission on Health (2003). Achieving the Promise: Transforming Mental Health Care in America. University of Michigan Library: Ann Arbor, MI.

Reed R, Harrow M, Herbener E, Martin E (2002). Executive function in schizophrenia: is it linked to psychosis and poor life functioning? Journal of Nervous and Mental Disease 190, 725-732.

Remington G, Kapur S (2010). Antipsychotic dosing: how much but also how often? Schizophrenia Bulletin 36, 900-903.

Samaha AN, Seeman P, Stewart J, Rajabi H, Kapur S (2007). 'Breakthrough' dopamine supersensitivity during ongoing antipsychotic treatment leads to treatment failure over time. Journal of Neuroscience 27, 2979-2986.

Stephens J, Pascal R, McHugh P (1997). Long-term follow-up of patients hospitalized for schizophrenia, 1913 to 1940. Journal of Nervous and Mental Disorders 185, 715-721.

Strauss J, Carpenter W (1972). The prediction of outcome in schizophrenia: I. Characteristics of outcome. Archives of General Psychiatry 27, 739-746.

Tandon R, Belmaker R, Gattaz WF (2008). World Psychiatric Association Pharmacopsychiatry Section statement on comparative effectiveness of antipsychotics in the 
treatment of schizophrenia. Schizophrenia Research 100, 20-38.

Vaillant G (1978). A 10-year follow-up of remitting schizophrenics. Schizophrenia Bulletin 4, 78-85.

Ventura J, Subotnik KL, Guzik LH, Hellemann GS, Gitlin MJ, Wood RC, Nuechterlein KH (2011). Remission and recovery during the first outpatient year of the early course of schizophrenia. Schizophrenia Research 132, 18-23.

Webber MA, Marder SR (2008). Better pharmacotherapy for schizophrenia: what does the future hold? Current Psychiatry Reports 10, 352-358.
Wechsler D (1955). Weschler Adult Intelligence Scale Manual. Psychological Corporation: New York.

White C, Stirling J, Hopkins R, Morris J, Montague L, Tantam D, Lewis S (2009). Predictors of 10-year outcome of first-episode psychosis. Psychological Medicine 39, 1447-1456.

Zigler E, Glick M (2001). The developmental approach to adult psychopathology. Clinical Psychologist 54, 2-11.

Zigler E, Levine J (1983). Hallucinations vs. delusions : a developmental approach. Journal of Nervous and Mental Disease 171, 141-146. 